\title{
Systemically administered neurotensin receptor agonist produces antinociception through activation of spinally projecting serotonergic neurons in the rostral ventromedial medulla
}

\author{
Yaqun $\mathrm{Li}^{1, *}$, Dong Ho Kang ${ }^{1, *}$, Woong Mo Kim ${ }^{1,2}$, Hyung Gon Lee ${ }^{1,3}$, Seung Hoon $\mathrm{Kim}^{1}$, Hyun Eung You ${ }^{2}$, \\ Jeong II Choi ${ }^{1,3}$, and Myung Ha Yoon ${ }^{1,3}$ \\ 'Department of Anesthesiology and Pain Medicine, Chonnam National University Hospital, Chonnam National University Medical School, \\ Gwangju, Korea \\ 2Department of Anesthesiology and Pain Medicine, Chonnam National University Hwasun Hospital, Hwasun, Korea \\ ${ }^{3}$ Center for Creative Biomedical Scientists, Chonnam National University Medical School, Gwangju, Korea
}

Received October 28, 2020

Revised December 12, 2020

Accepted December 16, 2020

Handling Editor: Younghoon Jeon

\section{Correspondence}

Woong Mo Kim

Department of Anesthesiology and Pain Medicine, Chonnam National University Medical School, 160 Baekseo-ro, Donggu, Gwangju 61469, Korea

Tel: +82-62-220-6895

Fax: +82-62-232-6294

E-mail: kimwm@jnu.ac.kr

\section{Hyung Gon Lee}

Department of Anesthesiology and Pain Medicine, Chonnam National University Medical School, 160 Baekseo-ro, Donggu, Gwangju 61469, Korea

Tel: +82-62-220-6895

Fax: +82-62-232-6294

E-mail: leehg@jnu.ac.kr

*These authors contributed equally to this work.
Background: Supraspinal delivery of neurotensin (NTS), which may contribute to the effect of a systemically administered agonist, has been reported to be either pronociceptive or antinociceptive. Here, we evaluated the effects of systemically administered NTSR1 agonist in a rat model of neuropathic pain and elucidated the underlying supraspinal mechanism.

Methods: Neuropathic pain was induced by L5 and L6 spinal nerve ligation in male Sprague-Dawley rats. The effects of intraperitoneally administered NTSR1 agonist PD 149163 was assessed using von Frey filaments. To examine the role of 5-HT neurotransmission, a serotonin (5-HT) receptor antagonist dihydroergocristine was pretreated intrathecally, and spinal microdialysis studies were performed to measure the change in extracellular level of 5-HT in response to PD 149163 administration. To investigate the supraspinal mechanism, NTSR1 antagonist 48692 was microinjected into the rostral ventromedial medulla (RVM) prior to systemic PD 149163. Additionally, the effect of intrathecal DHE on intra-RVM PD 149163 was assessed.

Results: Intraperitoneally administered PD 149163 exhibited a dose-dependent attenuation of mechanical allodynia. This effect was partially reversed by intrathecal pretreatment with dihydroergocristine and was accompanied by an increased extracellular level of 5-HT in the spinal cord. The PD 149163-produced antinociception was also blocked by intra-RVM SB 48692. Direct injection of PD 149163 into the RVM mimicked the maximum effect of the same drug delivered intraperitoneally, which was reversed by intrathecal dihydroergocristine.

Conclusions: These observations indicate that systemically administered NTSR1 agonist produces antinociception through the NTSR1 in the RVM, activating descending serotonergic projection to release 5-HT into the spinal dorsal horn.

Key Words: Analgesia; Central Nervous System; Microdialysis; Neuralgia; Neurotensin; Rats; Receptors, Neurotensin; Serotonin; Spinal Cord. (a) This is an open-access article distributed under the terms of the Creative Commons Attribution Non-Commercial License (http://creativecommons.org/licenses/by-nc/4.0/), which permits unrestricted non-commercial use, distribution, and reproduction in any medium, provided the original work is properly cited.

(c) The Korean Pain Society, 2021
Author contributions: Yaqun Li: Investigation; Dong Ho Kang: Writing/ manuscript preparation; Woong Mo Kim: Investigation; Hyung Gon Lee: Writing/manuscript preparation; Seung Hoon Kim: Data curation; Hyun Eung You: Writing/manuscript preparation; Jeong II Choi: Writing/manuscript preparation; Myung Ha Yoon: Funding acquisition. 


\section{INTRODUCTION}

Neurotensin (NTS) is a 13-amino acid neuropeptide neurotransmitter expressed in the peripheral organs and central nervous system (CNS) [1]. Peripherally, NTS acts as a paracrine and endocrine modulator of cardiovascular function and gastrointestinal motility in the heart, liver, lung, and digestive tract [2]. In the CNS, it acts as a primary neurotransmitter and a modulator of other molecules including dopamine, glutamate, GABA, acetylcholine, and serotonin (5-hydroxytriptamine, 5-HT), which suggest that NTS participates in the several pathophysiology of CNS diseases such as Parkinson's disease, schizophrenia, and pain [3].

There are three characterized receptors mediating the effects of NTS in the CNS; high affinity NTS1 receptor (NTSR1), low affinity NTS receptor (NTSR2), and intracellular NTS receptor (NTSR3) [4]. Among them, NTSR1, a Gprotein coupled receptor, is broadly expressed in cell bodies in the structures of the basal forebrain area and rostral ventromedial medulla (RVM), as well as in the substantia gelatinosa of the spinal cord and dorsal root ganglion $[5,6]$. These results indicate a possible antinociceptive role of NTS, mediated by supraspinal and spinal mechanisms. Intrathecal delivery of a selective NTSR1 agonist attenuated pain behavior in the rat model of inflammatory [7] and neuropathic pain [8], which were blocked by a selective antagonist of NTSR1. Thus, the antinociceptive action of NTSR1 and NTS by intrathecal delivery are clearly demonstrated in the literature. On the other hand, supraspinal action of NTS, which may contribute to the effect of a systemically administered agonist, has been reported to be either pronociceptive or antinociceptive. Intracerebroventricular injection of NTS at higher doses ( $\geq 100 \mathrm{ng}$ ) decreased acute thermal nociception, however, at lower doses $(25 \mathrm{ng})$, it antagonized the analgesic effect of intrathecal morphine [9]. Microinjection of NTSR1 agonist into the nucleus raphe magnus (NRM) produced an antinociceptive effect in a tail-flick test [6]. On the contrary, delivery of NTS antagonist into the nucleus reticularis paragigantocellularis lateralis enhanced morphine antinociception, thus demonstrating the pain facilitating action of NTS [10].

These observations indicate that the supraspinal action of NTS exhibits not only inhibitory but also facilitatory effects on pain modulation, depending on the dose of administration and the distinct area of activation mediating descending facilitation or inhibition of pain transmission $[11,12]$. Consequently, the effects of systemically delivered NTSRl agonist may vary according to the amount of the substance reaching a specific brain area. However, the net effects of NTSRl activation by systemic administration and the underlying supraspinal mechanisms are not clearly defined yet.

In the present study, we evaluated the effects of systemically administered NTSR1 agonist in a rat model of neuropathic pain, and elucidated the underlying mechanism of action using pharmacological analyses of behavior and in vivo microdialysis study.

\section{MATERIALS AND METHODS}

\section{Animals}

The experimental protocol was reviewed and approved by the Institutional Animal Care and Use Committee of Chonnam National University (No. IACUC-H-2016-47). Totally, 125 adult male Sprague-Dawley rats with weights ranging from 260 to $320 \mathrm{~g}$ were used in the present study. Animals were housed in a room with a constant temperature $\left(22^{\circ} \mathrm{C}-23^{\circ} \mathrm{C}\right)$, with an alternating 12 -hour light/dark cycle, and free access to food and water. Neuropathic pain was evoked by L5 and L6 spinal nerve ligation (SNL), according to the method described previously [13]. Rats exhibiting an inability to flex the left hind limb, indicating L4 nerve damage, were excluded from the study. Animals displaying a $50 \%$ withdrawal threshold of $<4.0 \mathrm{~g}$ by postoperative day 5 were considered to be neuropathic. For an intrathecal administration of the experimental drugs, a polyethylene-10 tubing was implanted intrathecally under sevoflurane anesthesia, as described previously [14]. Seven days were allowed after the surgery for recovery.

\section{Drugs}

The following drugs were used in the current study: PD 149163 (Sigma-Aldrich; St. Louis, MO), a selective NTSR1 agonist, was dissolved in saline. SR 48692 (Tocris Cookson Ltd., Bristol, UK), a selective non-peptide NTSR1 antagonist, and dihydroergocristine (Tocris Cookson Ltd.), a 5 -HT receptor antagonist, were dissolved in $100 \%$ and $70 \%$ dimethyl sulfoxide (DMSO), respectively. Intrathecal administration of the experimental drugs was performed using a hand-driven, gear-operated syringe pump through an implanted catheter in a volume of $10 \mu \mathrm{L}$, followed by flushing the catheter with $10 \mu \mathrm{L}$ of saline.

\section{Behavioral and pharmacological analysis of systemic NTSR1 agonist effects}

Behavioral analysis was done using the von Frey test, measuring the mechanical withdrawal threshold, at which a positive response was assumed when brisk withdrawal or flinching of the paw was observed during or immediately 
after applying one of the filaments with logarithmically increasing stiffness $(0.4,0.7,1.2,2.0,3.6,5.5,8.5$, and $15.0 \mathrm{~g})$. Then, the $50 \%$ probability paw withdrawal threshold was calculated using the up and down method [15]. The cutoff value was $15 \mathrm{~g}$.

On the day of the experiment, animals were acclimatized in a box with a wire mesh floor for at least 20 minutes and randomly allocated into the experimental groups for intraperitoneal delivery of the experimental drug or vehicle solution. Behavioral testing was carried out by an investigator blinded to the treatments. To investigate the effect of systemically administered NTSR1 agonist on pain behavior, PD $149163(10,30,100$, or $300 \mu \mathrm{g} / \mathrm{kg}, \mathrm{n}=6$ for each group) was administered intraperitoneally. Mechanical withdrawal thresholds were measured at 30,60 , $90,120,150$, and 180 minutes after the drug administration. To elucidate the role of the 5-HT signaling in NTSR1 agonist effect, non-selective 5-HT receptor antagonist dihydroergocristine $(30 \mu \mathrm{g}, \mathrm{n}=6)$ was injected 15 minutes prior to the delivery of NTSR1 agonist. The doses of the administered drugs were chosen based on a previous report [16].

\section{In vivo microdialysis study and HPLC quantification of spinal 5-HT release}

For assessing the effect of systemically administered NTSR1 agonist on spinal release of 5-HT, microdialysis studies were performed as described previously [17]. The rats were anesthetized with sevoflurane in $100 \%$ oxygen. The right femoral vein was cannulated for intravenous administration of lactated Ringer's solution at a rate of $1 \mathrm{~mL} /$ $\mathrm{hr}$. The rectal temperature was controlled at $37^{\circ} \mathrm{C}-38^{\circ} \mathrm{C}$ with a heating pad placed under the abdomen. After performing laminectomy at the level of the T13-L1 vertebrae, and subsequent exposure of the L3 to L5 segment of the spinal cord, the dural surface was covered with mineral oil and the rat was placed in a stereotaxic holder. After opening the dura, a microdialysis probe (outer diameter, 0.22 $\mathrm{mm}$; inner diameter, $0.20 \mathrm{~mm}$; length, $1 \mathrm{~mm}$; Eicom Co., Kyoto, Japan) was inserted just lateral to the dorsal root into the dorsal horn by advancing at an angle of $30^{\circ}$ and to a depth of $1 \mathrm{~mm}$ using a micromanipulator (model MM3; Narishige, Tokyo, Japan). The probe was perfused with Ringer's solution $(147.0 \mathrm{mmol} / \mathrm{L} \mathrm{NaCl}, 4.0 \mathrm{mmol} / \mathrm{L} \mathrm{KCl}$, and $\left.2.3 \mathrm{mmol} / \mathrm{L} \mathrm{CaCl}{ }_{2}\right)$ at a constant rate $(1 \mu \mathrm{L} / \mathrm{min})$ using a microsyringe pump (ESP-64; Eicom Co.). After 120 minutes of constant perfusion, two samples were consecutively collected to determine the basal 5-HT concentrations in the dialysate [18]. To measure the change of 5-HT level by systemically injected NTSR1 agonist, $300 \mu \mathrm{g} / \mathrm{kg}$ of PD $149163(\mathrm{n}=5)$ was injected intraperitoneally. Thereafter, the 15-minute fractions of perfusate were collected into an autoinjector (EAS-20; Eicom Co.).

The samples $(15 \mu \mathrm{L})$ were injected automatically into the HTEC-500 system (Eicom Co.) to analyze the 5-HT concentrations by high-performance liquid chromatography (HPLC) with electrochemical detection. The chromatographic conditions were as follows. The mobile phase comprised $0.1 \mathrm{~mol} / \mathrm{L}$ ammonium acetate buffer $(\mathrm{pH}$ 6.0), methanol (7:3 vol/vol) containing $0.05 \mathrm{~mol} / \mathrm{L}$ sodium sulfonate, and $50 \mathrm{mg} / \mathrm{L}$ EDTA-2Na. The column was an EICOMPAC CAX $(2.0 \times 200.0 \mathrm{~mm}$; Eicom Co. $)$. The working electrode was glassy carbon (WE-3G; Eicom Co., flow rate $0.25 \mathrm{~mL} / \mathrm{min}$ ). The detector voltage and temperature were set at $0.45 \mathrm{~V}$ and $35.0^{\circ} \mathrm{C}$, respectively. The retention time for $5-\mathrm{HT}$ was 13.1 minutes and the detection limit was $30 \mathrm{fg}$ per injection.

\section{Intracranial microinjection study}

To investigate the role of RVM NTSR1 in the antinociceptive effect of NTSRl agonist, intracranial RVM cannulation was performed by a stereotaxic surgery according to coordinates derived from the brain atlas of Paxinos and Watson [19]. Under sevoflurane anesthesia, a 26-gauge stainless steel guide cannula (Plastics One, Roanoke, VA) was directed just dorsal to the NRM of RVM (anteroposterior, Bregma $-11 \mathrm{~mm}$; mediolateral, midline $0.0 \mathrm{~mm}$; dorsoventral, skull -9 mm). Cannulae were secured to the skull by acrylic dental cement and stainless-steel screws, and were fitted with a solid 25-gauge stainless steel obturator (Plastics One). Animals were allowed to recover for 7 days. Intracranial microinjections of experimental drugs $(0.5 \mu \mathrm{L})$ were performed using injectors extended $1 \mathrm{~mm}$ beyond the tip of the implanted guide cannula, at a rate of $0.2 \mu \mathrm{L} / \mathrm{min}$, using a microsyringe pump (ESP-101; Eicom Co.).

On the day of the experiment, animals were acclimatized in the experimental room as described above. To determine whether the systemic administration of NTSR1 agonist acts through the receptors in the NRM, a selective antagonist SR $48692(10 \mu \mathrm{g}, \mathrm{n}=5)$ was delivered via the implanted intracranial cannula 10 minutes prior to the intraperitoneal injection of NTSR1 agonist, and von Frey tests were performed thereafter. The behavioral effect of NTSR1 activation in the NRM was further tested by microinjection of PD $149163(5,10,20 \mathrm{ng}, \mathrm{n}=5$ for each group) through the cannula. Finally, to elucidate the role of descending serotonergic projection to the spinal cord from NRM in the NTSR1 signaling, dihydroergocristine (30 $\mu \mathrm{g}, \mathrm{n}=5$ ) was intrathecally administered 10 minutes prior to the intra-NRM injection of PD $149163(n=5)$. The doses of the administered drugs were chosen based on pilot ex- 



Fig. 1. Time-response (A) and dose-response (B) data showing the effects of intraperitoneally administered PD 149163, on the hind paw withdrawal response in spinal nerve-ligated rats. Data are presented as the mechanical withdrawal thresholds in grams or the area under the time course curve (AUC) for the withdrawal threshold. Each line or bar represents the mean \pm standard error of mean of 6 rats. i.p.: intraperitoneal, BL: baseline value, i.t.: intrathecal, DHE: dihydroergocristine. ${ }^{*} P=0.048$ compared to the vehicle group. ${ }^{\S} P<0.001$ compared to the vehicle group. ${ }^{\dagger} P=0.032$ compared to the PD $149163300 \mu \mathrm{g} / \mathrm{kg}$ group.

periments.

After testing, animals were decapitated and the brains were rapidly removed and immersed in $10 \%$ formalin for 24 hours and then in $30 \%$ sucrose for 48 hours. The tissues were frozen sectioned for verification of the cannulation site, using microscopic examination. Data from animals with misplaced cannulas were removed from the analyses.

\section{Statistical analysis}

All data are shown as mean \pm standard error of mean. The time-response data are exhibited as the withdrawal threshold in grams. The dose-response data are quantified as the area under the time course curves (AUC) for each dose using the trapezoidal rule, which was analyzed by one-way analysis of variance (ANOVA) with the Bonferroni post hoc test. The antagonistic effects of PD 149163 were compared using an unpaired $t$-test. For these parametric techniques, the Kolmogorov-Smirnov test for assessing the normality of the distribution of data and Levene's test for equality of variances were performed. When the assumption of equal variance was violated, a compensated $t$-value and Welch's statistics with the Games-Howell post hoc test was presented for the $t$-test and ANOVA, respectively. For the microdialysis data, the Friedman test, with a Wilcoxon signed rank test with the Bonferroni adjustment for post hoc testing, was performed. All statistical analyses were performed using PASW Statistics software (version 18.0; IBM Co., Armonk, NY) and $P$ values $<0.05$ were considered to indicate statistical significance.

\section{RESULTS}

\section{Systemic administration of NTSRl agonist exhibits an antiallodynic effect in a dose-dependent manner}

SNL resulted in a characteristic mechanical allodynia (Fig. 1A). The baseline values did not differ among the groups. Intraperitoneal administration of PD 149163 increased the mechanical withdrawal thresholds to von Frey stimulation in a dose dependent manner (Fig. 1). A one-way ANOVA of the AUCs of time-response curves revealed a statistically significant differences among the groups with the vehicle and different doses of PD 149163 treatments, Welch's $F$ (4, $11.99)=110.96, P<0.001$. Post-hoc comparisons using the Games-Howell procedure indicated that the AUCs for the PD 149163100 and $300 \mu \mathrm{g} / \mathrm{kg}$ groups were significantly different from that of the vehicle-treated control group, with $P=0.048$ and $<0.001$, respectively.

\section{Spinal release of 5-HT mediates NTSR1 agonist- produced antinociception}

The antiallodynic effect of intraperitoneal PD 149163 (300 $\mu \mathrm{g} / \mathrm{kg}$ ) was attenuated by intrathecal pretreatment with a non-selective 5HT receptor antagonist dihydroergocristine $(P=0.032$, Fig. 1B). For assessing the effect of systemic NTSR1 agonist on spinal 5-HT release, a microdialysis study was performed to measure the change of extracellular concentration of 5-HT in the spinal dorsal horn. The baseline levels of extracellular 5-HT between the groups of saline and PD 149163 treatments did not significantly dif- 
fer; $232.16 \pm 83.83 \mathrm{fg} / \mu \mathrm{L}$ and $143.93 \pm 24.34 \mathrm{fg} / \mu \mathrm{L}$, respectively, $P=0.350$. The Friedman test revealed a statistically significant difference in 5-HT level across time periods in the PD 149163 group, $\chi^{2}(4, \mathrm{n}=5)=4, P=0.019$. Significant differences compared to baseline were shown at 15, 30, and 45 minutes of PD 149163 administration, by post hoc testing with the Wilcoxon signed rank test $(P=0.009,0.009$, and 0.010, respectively, Fig. 2).

\section{Involvement of RVM in NTSR1 agonist-produced antinociception}

To determine whether the RVM participates in the effect



Fig. 2. Microdialysis measurements of spinal 5-HT following intraperitoneal administration of PD $149163(300 \mu \mathrm{g} / \mathrm{kg})$ or vehicle. Data are presented over time as a mean \pm standard error of mean percentage of the baseline ( $n=5$ in each group). BL: baseline value. ${ }^{*} P<0.05$ compared to baseline value. of systemic NTSR1 agonist, a selective NTSR1 antagonist, SR 48692, was microinjected into the NRM 10 minutes prior to the delivery of PD 149163. SR 48692 administered alone into the NRM did not affect baseline pain behavior. And neither did the vehicle DMSO affect the effect of PD 149163. The antinociception produced by intraperitoneal administration of PD $149163(300 \mu \mathrm{g} / \mathrm{kg})$ was significantly reduced by pretreatment with intra-NRM SR $48692(10 \mu \mathrm{g}$, $P=0.002$, Fig. 3).

\section{Involvement of descending serotonergic projection to the spinal cord from RVM}

To further investigate the role of RVM NTSRl and serotonergic projection to the spinal cord, NTSRl agonist was directly injected into the NRM with or without pretreatment with intrathecal 5-HT antagonist. Intra-NRM microinjection of PD 149163 increased mechanical withdrawal thresholds to von Frey stimulation (Fig. 4). A one-way ANOVA of AUCs of time-response curves revealed a statistically significant difference among the groups, $F(3,16)=$ $12.40, P<0.001$. Post-hoc comparisons using the Bonferroni correction indicated that the AUCs for 5, 10, and $20 \mathrm{ng}$ groups of PD 149163 were significantly greater compared to that of the control group $(P=0.005,001$, and $<0.001$, respectively). However, the AUCs did not differ among the groups of different doses. Intrathecal pretreatment with dihydroergocristine significantly reduced the antiallodynic effect of intra-NRM PD 149163 (5 ng, $P=0.048$, Fig. 4B).

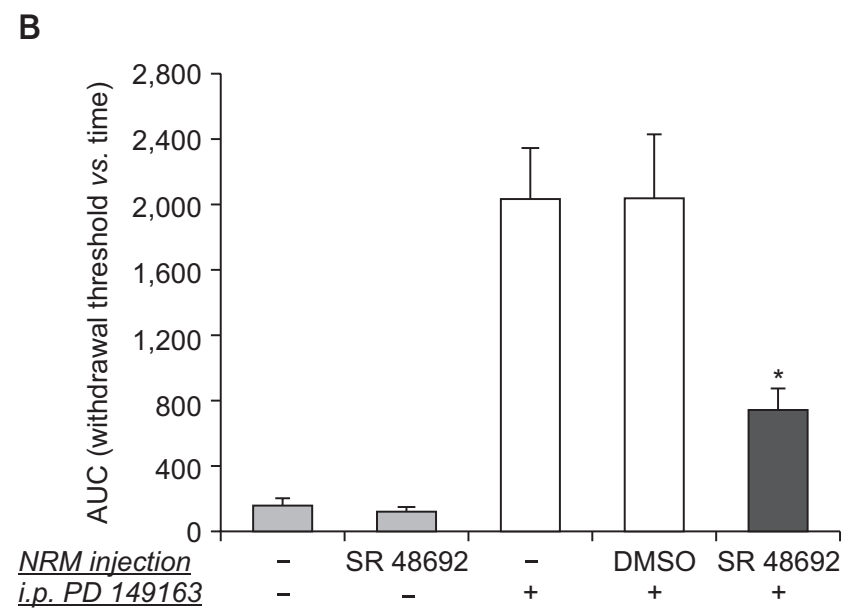

Fig. 3. Time-response data (A) and quantification by area under the time course curves (AUC) (B) showing the effects of microinjection of SB 48692 or vehicle into the nucleus raphe magnus (NRM) and intraperitoneal administration of PD 149163 or vehicle. Data are presented as the mechanical withdrawal thresholds in grams or the AUC for the withdrawal threshold. Each line or bar represents the mean \pm standard error of mean of 5 rats. inj.: injection, i.p.: intraperitoneal, BL: baseline value, DMSO: dimethyl sulfoxide. ${ }^{*} P=0.002$ compared to the intraperitoneal PD $149163 \mathrm{group}(300 \mu \mathrm{g} / \mathrm{kg}$ ). 

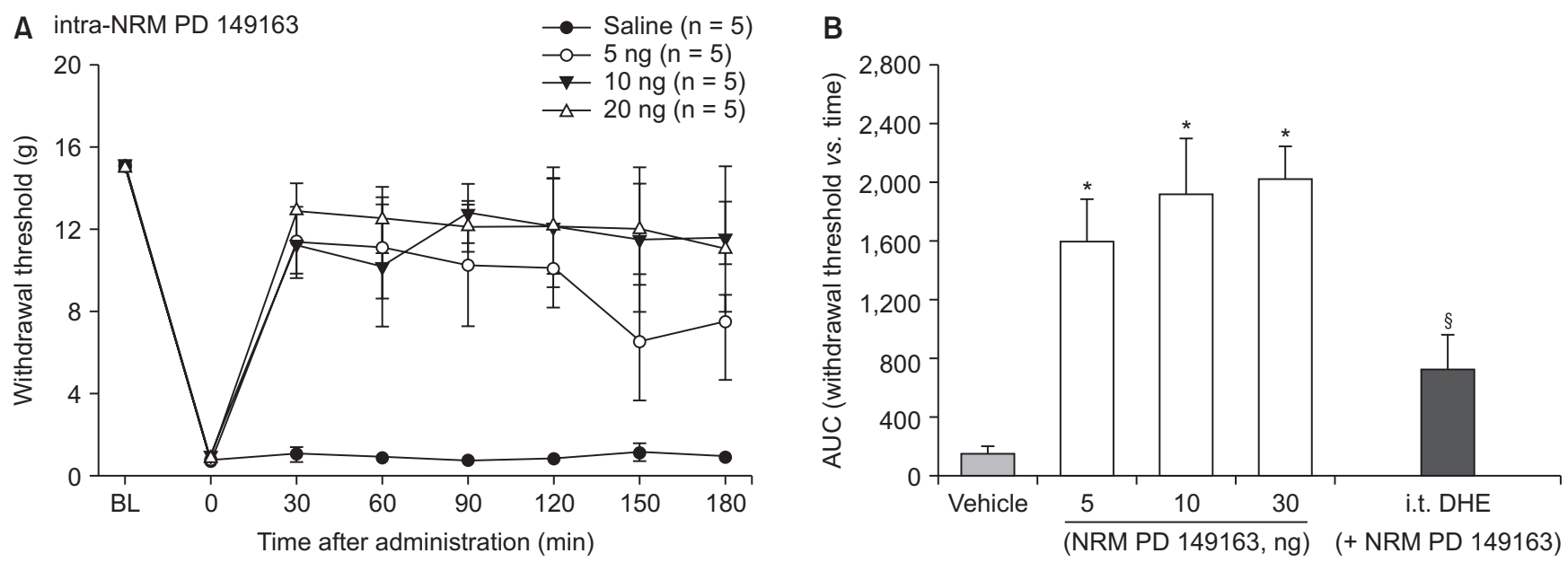

Fig. 4.Time-response (A) and dose-response (B) data showing the effects of intra-nucleus raphe magnus (NRM) administration of PD 149163 , on the hind paw withdrawal response in spinal nerve-ligated rats. Data are presented as the mechanical withdrawal thresholds in grams or the area under the time course curve (AUC) for the withdrawal threshold. Each line or bar represents the mean \pm standard error of mean of 5 rats. BL: baseline value, i.t.: intrathecal, DHE: dihydroergocristine. ${ }^{*} P<0.005$ compared to the vehicle group. ${ }^{\S} P=0.048$ compared to the PD $1491635 \mathrm{ng}$ group.

\section{DISCUSSION}

The present study demonstrated that intraperitoneally administered PD 149163 exhibited a dose-dependent attenuation of mechanical allodynia in a rat model of neuropathic pain. The antiallodynic effect of systemic PD 149163 was partially reversed by intrathecal pretreatment with dihydroergocristine, and was accompanied by an increased extracellular level of 5-HT in the spinal dorsal horn. The antinociception produced by intraperitoneal administration of PD 149163 was dependent on NTSR1 in the NRM of the RVM. Direct injection of PD 149163 into the NRM mimicked the maximum effect of the same drug delivered intraperitoneally, which was reversed by intrathecal dihydroergocristine. Taken together, these observations indicate that systemically administered NTSR1 agonist produces antinociception through the NTSR1 in the RVM, activating descending serotonergic projection to release 5-HT into the spinal dorsal horn.

The present study shows that systemic or intra-RVM injection of NTSR1 agonist produces a dose-dependent antinociceptive effect, in contrast to the previous studies reporting bipolar actions. This discrepancy may result from receptor selectivity of the chemicals and the diversity in the pathophysiology of the animal model used. Bipolar actions on pain modulation was reported when NTS peptide, rather than a selective agonist of NTSR1, was administered cerebroventricularly or intracranially in an acute nociception model [9-12]. Because multiple NTSRs with varying degree of affinity exist, a non-selective agonist NTS peptide may activate either facilitatory or inhibitory pathways of pain modulation through distinct receptor subtypes, depending on the dose of administration $[4,11]$. This find- ing suggests that the role of endogenous NTS is in maintaining homeostatic balance in the individual response to pain by acting biphasically. Contrarily, we could assume that a selective activation of NTSR1, in the current study, might have produced a dose-dependent antinociception in a model of pathologic pain. Additionally, the bipolar effects of NTS may depend on the intensity of nociceptive stimulation. In a study by Bodnar et al. [20], intracerebroventricularly delivered NTS antiserum produced either hyperalgesia or antinociception, depending on the degree of noxious stimuli.

In the present study, systemically administered PD 149163 enhanced the release of 5-HT into the extracellular space in the spinal cord, which was revealed by an in vivo microdialysis study. The antinociception produced by the same drug was attenuated by an intrathecal blockade of 5-HT receptors. Therefore, the increased extracellular level of 5-HT by PD 149163 might have contributed to this antinociceptive effect. Based on the observation that virtually all the serotonergic fibers projecting to the spinal cord arise from the brain [21], we could assume that intraperitoneally administered PD 149163 acts supraspinally to increase spinal release of 5-HT.

A majority of serotonergic innervation to the spinal cord arises within the vicinity of the RVM, which has been known as an important supraspinal site of the descending pain modulatory system [21,22], although a small proportion of serotonergic fibers from the dorsal raphe nucleuswhich predominantly innervates the forebrain-modestly contributes to the spinal projection [23]. Among the subdivisions of the RVM, NRM is a structure rich in serotonergic neurons projecting to the superficial laminae of the spinal dorsal horn to modulate pain transmission [21]. There- 
fore, we performed intra-NRM microinjection study to elucidate supraspinal mechanism of systemic PD 149163. This study clearly demonstrated that the antinociception produced by systemic PD 149163 was reversed by blockade of NTSR1 expressed in the NRM. Furthermore, the behavioral effect of activation of NRM NTSR1 by microinjection of PD 149163 was attenuated by intrathecal pretreatment with 5-HT receptor antagonist. These results clearly indicate that systemically administered NTSRl agonist acts on the NTSR1 in the NRM of the RVM to activate descending serotonergic neurons projecting to the spinal dorsal horn cord, consequently releasing 5 -HT into the extracellular space to produce antinociception.

The observations from the current study are consistent with previous reports. Buhler et al. [6] demonstrated by immunohistochemical technique that NTSR1 is co-localized in the serotonergic neurons, projecting to the spinal cord from the NRM. Intra-RVM injections of NTS or selective NTSR1 agonist increased the tail flick latency that was blocked by pretreatment with intrathecal 5-HT receptor antagonist $[6,24]$. These observations raised a possible role for descending serotonergic neurons in the RVM in NTSR1mediated antinociception. The present study provides the first verification of this pathway by administrating NTSR1 agonist systemically and by identifying the change in extracellular level of 5-HT using in vivo microdialysis study.

In contrast to the current study, in the chemotherapy-induced peripheral neuropathy (CIPN) model, the antinociceptive effect of systemically administered NTSR1 agonist was not suppressed by an intrathecal blockade of 5-HT receptor antagonist [16]. This discrepancy may result from the differences in the experimental pain model, behavioral test, and the species used. For example, a recent study using a rat model of CIPN reported increased activation of serotonergic RVM neurons and increased expression of 5 -HT at the superficial dorsal horn together with up-regulation of pronociceptive 5-HT3 receptor, indicating a facilitatory rather than an inhibitory role for the descending serotonergic transmission in CIPN [25]. Therefore, differences in the neurochemical and functional organization of the nociceptive pathways in CIPN from that in the nerve injury-induced neuropathic pain model used for the current study might have contributed to this inconsistency.

There are several limitations to the present study. The antiallodynic effects of both intraperitoneal and intraNRM PD 149163 were only partially reversed by intrathecal pretreatment with dihydroergocristine. Therefore, there may be other mechanisms that underlie the antinociceptive effect of NTSR1 agonist. For example, the expression of NTSR1 in the spinal dorsal horn and the antinociceptive effect of intrathecally delivered NTSR1 was reported $[5,8]$. In addition, the antinociceptive action of NTS was inhib- ited by the depletion of spinal norepinephrine [26]. Therefore, the roles of spinal NTSR1 and noradrenergic neurotransmission remain to be investigated in future studies. We need to consider that the present study utilized a small sample size which may lead to a low power, increasing false positives, and overestimated effect sizes.

In conclusion, the current study demonstrated that systemically administered NTSR1 agonist produces antinociception through the NTSR1 in the RVM, activating descending serotonergic projection to release 5 -HT into the spinal dorsal horn.

\section{CONFLICT OF INTEREST}

No potential conflict of interest relevant to this article was reported.

\section{FUNDING}

This study was supported by a grant from Basic Science Research Program through the National Research Foundation of Korea (NRF) funded by the Ministry of Education (NRF-2019R1I1A3A01063969), South Korea.

\section{ORCID}

Yaqun Li, https://orcid.org/0000-0002-3073-0165

Dong Ho Kang, https://orcid.org/0000-0002-5299-6861

Woong Mo Kim, https://orcid.org/0000-0002-3523-7468

Hyung Gon Lee, https://orcid.org/0000-0003-4898-4355

Seung Hoon Kim, https://orcid.org/0000-0003-0579-6522

Hyun Eung You, https://orcid.org/0000-0002-3307-8563

Jeong Il Choi, https://orcid.org/0000-0002-3571-2599

Myung Ha Yoon, https://orcid.org/0000-0002-9962-0255

\section{REFERENCES}

1. Carraway R, Leeman SE. The isolation of a new hypotensive peptide, neurotensin, from bovine hypothalami. J Biol Chem 1973; 248: 6854-61.

2. Mustain WC, Rychahou PG, Evers BM. The role of neurotensin in physiologic and pathologic processes. Curr Opin Endocrinol Diabetes Obes 2011; 18: 75-82.

3. Boules M, Li Z, Smith K, Fredrickson P, Richelson E. Diverse roles of neurotensin agonists in the central nervous system. Front Endocrinol (Lausanne) 2013; 4: 36.

4. Kleczkowska P, Lipkowski AW. Neurotensin and neurotensin receptors: characteristic, structure-activity relationship and 
pain modulation--a review. Eur J Pharmacol 2013; 716: 54-60.

5. Fassio A, Evans G, Grisshammer R, Bolam JP, Mimmack M, Emson PC. Distribution of the neurotensin receptor NTS1 in the rat CNS studied using an amino-terminal directed antibody. Neuropharmacology 2000; 39: 1430-42.

6. Buhler AV, Choi J, Proudfit HK, Gebhart GF. Neurotensin activation of the NTR1 on spinally-projecting serotonergic neurons in the rostral ventromedial medulla is antinociceptive. Pain 2005; 114: 285-94.

7. Roussy G, Dansereau MA, Doré-Savard L, Belleville K, Beaudet N, Richelson E, et al. Spinal NTS1 receptors regulate nociceptive signaling in a rat formalin tonic pain model. J Neurochem 2008; 105: 1100-14.

8. Guillemette A, Dansereau MA, Beaudet N, Richelson E, Sarret P. Intrathecal administration of NTS1 agonists reverses nociceptive behaviors in a rat model of neuropathic pain. Eur J Pain 2012; 16: 473-84.

9. Holmes BB, Rady JJ, Smith DJ, Fujimoto JM. Supraspinal neurotensin-induced antianalgesia in mice is mediated by spinal cholecystokinin. Jpn J Pharmacol 1999; 79: 141-9.

10. Urban MO, Smith DJ. Localization of the antinociceptive and antianalgesic effects of neurotensin within the rostral ventromedial medulla. Neurosci Lett 1994; 174: 21-5.

11. Smith DJ, Hawranko AA, Monroe PJ, Gully D, Urban MO, Craig CR, et al. Dose-dependent pain-facilitatory and -inhibitory actions of neurotensin are revealed by SR 48692, a nonpeptide neurotensin antagonist: influence on the antinociceptive effect of morphine. J Pharmacol Exp Ther 1997; 282: 899-908.

12. Urban MO, Coutinho SV, Gebhart GF. Biphasic modulation of visceral nociception by neurotensin in rat rostral ventromedial medulla. J Pharmacol Exp Ther 1999; 290: 207-13.

13. Kim SH, Chung JM. An experimental model for peripheral neuropathy produced by segmental spinal nerve ligation in the rat. Pain 1992; 50: 355-63.

14. Koh GH, Song H, Kim SH, Yoon MH, Lim KJ, Oh SH, et al. Effect of sec-O-glucosylhamaudol on mechanical allodynia in a rat model of postoperative pain. Korean J Pain 2019; 32: 87-
96.

15. Chaplan SR, Bach FW, Pogrel JW, Chung JM, Yaksh TL. Quantitative assessment of tactile allodynia in the rat paw. J Neurosci Methods 1994; 53: 55-63.

16. Yin M, Kim YO, Choi JI, Jeong S, Yang SH, Bae HB, et al. Antinociceptive role of neurotensin receptor 1 in rats with chemotherapy-induced peripheral neuropathy. Korean J Pain 2020; 33: 318-25.

17. Lee HG, Choi JI, Yoon MH, Obata H, Saito S, Kim WM. The antiallodynic effect of intrathecal tianeptine is exerted by increased serotonin and norepinephrine in the spinal dorsal horn. Neurosci Lett 2014; 583: 103-7.

18. Chae JW, Kang DH, Li Y, Kim SH, Lee HG, Choi JI, et al. Antinociceptive effects of nefopam modulating serotonergic, adrenergic, and glutamatergic neurotransmission in the spinal cord. Neurosci Lett 2020; 731: 135057.

19. Paxinos G, Watson C. Paxinos and Watson's the rat brain in stereotaxic coordinates. 7th ed. London, Academic Press. 2014, pp 116-32.

20. Bodnar RJ, Wallace MM, Nilaver G, Zimmerman EA. The effects of centrally administered antisera to neurotensin and related peptides upon nociception and related behaviors. Ann N Y Acad Sci 1982; 400: 244-58.

21. Millan MJ. Descending control of pain. Prog Neurobiol 2002; 66: 355-474.

22. Mason P. Central mechanisms of pain modulation. Curr Opin Neurobiol 1999; 9: 436-41.

23. Wang QP, Nakai Y. The dorsal raphe: an important nucleus in pain modulation. Brain Res Bull 1994; 34: 575-85.

24. Fang FG, Moreau JL, Fields HL. Dose-dependent antinociceptive action of neurotensin microinjected into the rostroventromedial medulla of the rat. Brain Res 1987; 420: 171-4.

25. Costa-Pereira JT, Serrão P, Martins I, Tavares I. Serotoninergic pain modulation from the rostral ventromedial medulla (RVM) in chemotherapy-induced neuropathy: the role of spinal 5-HT3 receptors. Eur J Neurosci 2020; 51: 1756-69.

26. Behbehani MM. Physiological mechanisms of the analgesic effect of neurotensin. Ann N Y Acad Sci 1992; 668: 253-65. 